\title{
GESTIÓN DE LA CALIDAD EN LA DISTRIBUCIÓN ELÉCTRICA DEL ESTADO ZULIA
}

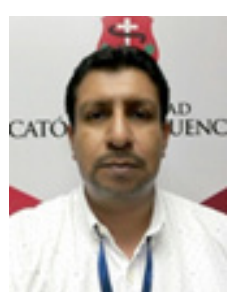

\author{
Orellana Orellana, Carlos ${ }^{1}$ \\ Universidad Católica de Cuencas. Ecuador. \\ corellanao@ucacue.edu.ec
}

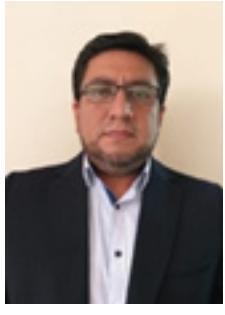

\section{Orellana Orellana, Edison Fernando ${ }^{2}$ \\ Universidad Católica de Cuencas. Ecuador. \\ eforellanao@ucacue.edu.ec}

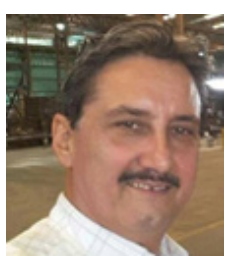

\section{Hernández Rincón, José Luis ${ }^{3}$ \\ Universidad del Zulia, Venezuela \\ hernandezjlk@hotmail.com}

\section{RESUMEN}

Se analiza la gestión de la calidad en la distribución eléctrica del estado Zulia, específicamente en el proceso de distribución eléctrica residencial e industrial. La investigación es descriptiva, con diseño de campo, transeccional y no experimental. La población estuvo determinada por el departamento de distribución eléctrica en los municipios de la Costa Oriental del Lago de la Corporación Eléctrica de Venezuela (CORPOELEC); los informantes claves fueron: gerentes de departamento, coordinadores de departamento y jefes de área. Para la población se realizó un censo. Se diseñó un instrumento de recolección de datos el cual contiene las preguntas relacionadas a la variable de estudio, dimensiones e indicadores. Este instrumento fue validado por cinco expertos y sometido a confiabilidad a través del método Alfa de Cronbach teniendo como resultado 0,968.

\footnotetext{
${ }^{1}$ Carlos Patricio Orellana Orellana

Magister en Administración de Empresas. Ingeniero Empresarial. Docente a tiempo completo de la Universidad Católica de Cuencas. Ecuador. Email: corellanao@ucacue.edu.ec

${ }^{2}$ Edison Fernando Orellana Orellana

Magister en Administración de Empresas. Ingeniero Empresarial. Director de la Carrera de Administración de Empresas. Universidad Católica de Cuencas. Ecuador. Email: eforellanao@ucacue.edu.ec

${ }^{3}$ José Luis Hernández Rincón

Magister en Gerencia de Empresas. Ingeniero Electricista. Universidad del Zulia. Email: hernandezjlk@hotmail.com
} 
Se empleó la estadística descriptiva presentando los resultados en tablas de frecuencias, para ello se utilizó el programa SPSS. La investigación estuvo soportada teóricamente por autores como: Gutiérrez (2010), Cantú (2006), Camisón y col (2007), Cuatrecasas (2010), entre otros. Entre los resultados se destacan: la dimensión procesos del sistema de gestión de la calidad, arrojó una media de 4.01, obteniendo un valor adecuado y en cuanto a los principios de gestión de la calidad, la media obtenida fue de 3.83 , siendo calificada como adecuada. Como conclusión se puede decir que tanto los procesos, como los principios se desarrollan adecuadamente, en la distribución eléctrica del estado Zulia.

Palabras clave: Gestión de la calidad, procesos, principios, distribución eléctrica.

\section{QUALITY MANAGEMENT IN THE ELECTRICITY DISTRIBUTION \\ OF THE ZULIA STATE \\ ABSTRACT}

The management of the quality in the electrical distribution of the Zulia state is analyzed, specifically in the residential and industrial electrical distribution process. The research is descriptive, with field design, transectional and non-experimental. The population was determined by the department of electrical distribution in the municipalities of the Eastern Coast of the Lake of the Electricity Corporation of Venezuela (CORPOELEC); The key informants were: department managers, department coordinators and area managers. A census was conducted for the population. A data collection instrument was designed which contains the questions related to the study variable, dimensions and indicators. This instrument was validated by five experts and subjected to reliability through the Cronbach's Alpha method, resulting in 0.968. Descriptive statistics was used, presenting the results in frequency tables, for which the SPSS program was used. The research was supported theoretically by authors such as: Gutiérrez (2010), Cantú (2006), Camisón y col (2007), Cuatrecasas (2010), among others. Among the results are: the process dimension of the quality management system, gave an average of 4.01, obtaining an adequate value and as for the principles of quality management, the average obtained was 3.83 , being rated as adequate . In conclusion, it can be said that both the processes and the principles are well developed in the electric distribution of the Zulia state.

Keywords: Quality management, processes, principles, electrical distribution. 


\section{INTRODUCCIÓN}

En la mayoría de los países en el mundo, al igual que en Venezuela, el movimiento por la gestión de la calidad es ahora evidentemente universal por lo que las organizaciones se ven en la necesidad estratégica de mejorarla cada día más. Este movimiento por la calidad nace y se acentúa en las dos primeras décadas del siglo XX en Estados Unidos de Norteamérica y se propagó en los años 40 y 50 en Japón para luego extenderse de forma mejorada en Occidente en los años 70 y expandiéndose por todo el mundo a partir de los años 80 . Como se indicó anteriormente la visión de la calidad fue predominante en Occidente hasta la década de 1970, sufriendo una evolución importante en las décadas siguientes y manteniendo su vigencia durante todo este tiempo.

Según Cantú (2006) la calidad es un término difícil de definir, ya que se mantiene en constante evolución; definirlo implica insertarlo en el contexto de la época en que fue desarrollado, los enfoques de calidad permiten entender sus diferentes definiciones, que van desde calidad en general hasta control de calidad, control estadístico, control total de calidad, aseguramiento de la calidad, calidad total y calidad Seis Sigma.

Por lo tanto, el concepto de calidad ha transitado por diversas eras: la de inspección (siglo XLX), la de control estadístico de procesos inmersa en la década de los treinta, la del aseguramiento de la calidad que abarcaba la década de los cincuenta y la época de la administración estratégica por calidad total que acompaño la década de los noventa, por eso desde sus inicios el hombre se ha interesado por la calidad, Al comienzo la calidad consistía en seleccionar alimentos y vestidos que le pudieran beneficiar de manera propia, no existían procesos inherentes a manufactura. El consumidor y el fabricante se conocían perfectamente, sus transacciones comerciales eran cara a cara, no existían especificaciones ni garantías, el productor era el único garante de la satisfacción estricta del cliente, la calidad de diseño se obtenía con mayor facilidad, era más sencillo lograr la calidad de conformancia y la satisfacción del consumidor.

En consecuencia, desde hace décadas los temas y procesos de investigación sobre la gestión de la calidad han constituido un factor necesario y obligatorio dentro del contorno empresarial, así como también un icono actual en los estudios académicos de la gran sociedad y a que representa un objeto concluyente para el progreso adecuado de las organizaciones, en virtud de todos los desafíos que actualmente deben afrentarse y más aún en este mundo global.

De esta manera la historia del movimiento de la calidad, permite identificar varias generaciones dentro de toda la literatura, con diferencia notable en su concepto de gestión de calidad. Estos enfoques han ido adecuándose a las nuevas concepciones de calidad, tanto en la determinación 
de la variable a incluir como de las relaciones existentes entre ellas, las condiciones filosóficas y la filosofía para su introducción. La complejidad ha sido además atizada con los procesos de reforma de todos los modelos existentes. Consecuentemente, no se dispone de una definición cabal y comúnmente aceptada de lo que se entiende por Gestión de Calidad, utilizándose una variedad de etiquetas o denominaciones para el mismo concepto.

En este sentido, la calidad ya no se limita actualmente a la calidad de un producto o servicio, si no que cubre todos los ámbitos a través de las cuales las organizaciones e instituciones satisfacen las necesidades y expectativas de sus clientes y consumidores, de su personal y de la sociedad en general, alcanzando la calidad total .Es por ello, que el concepto de calidad total se aplica, hoy en día, a todas las acciones y procesos que se despliegan dentro de las empresas; lo que pasa por incorporar una cultura de calidad a la gestión empresarial y administrativa. Así la implantación de un conjunto de principios dirigidos a elevar la eficacia y la productividad facilita y propicia una excelente gestión de la calidad.

Al respecto Cantú (2006) dice la calidad total es el soporte para la competitividad de las empresas en todo el mundo, esta se enfoca en considerar al conjunto de técnicas y procedimientos para la planeación, el control y el mejoramiento de todas las actividades organizacionales, con el objeto de entregar al cliente un producto o servicio que satisfaga sus necesidades y expectativas.

En este orden de ideas, la gestión de la calidad se ha convertido actualmente en una condición necesaria para cualquier estrategia dirigida hacia el éxito competitivo de las empresas y de las instituciones. En tal sentido, según Camisón y col (2007) el concepto de calidad ha evolucionado de forma importante durante el siglo XX, estando las primeras aportaciones orientadas básicamente a la aplicación de técnicas estadísticas para la inspección y el control de los productos y procesos industriales.

Como un aporte del maestro Edward W Deming, quien después de la segunda guerra mundial fue enviado a Japón a realizar estudios de censos y durante su estancia desarrollo considerables aportes al tema de calidad, confirió el Premio Deming a muchas empresas importantes y constituye el más alto galardón a la calidad en Japón, este además utilizo el CEP (Control Estadísticos de Procesos) en forma cada vez más avanzada, sino además desarrollo una forma de administración con participación, la cual aprovechaba los conocimientos y las habilidades de cada empleado, en todos los niveles, por medio de equipos humanos y sistemas de sugerencias y siempre con la mira puesta en el cliente, factor fundamental de las organizaciones. 
Las empresas llamaron a este nuevo sistema administrativo control total de calidad o control de calidad en toda la empresa. Las empresas no solamente se esforzaron por mejorar continuamente los procesos para reducir la variación o eliminar los defectos sino que también innovaron constantemente a fin de avanzar más. Por lo tanto las organizaciones en la actualidad aplican sistemas de gestión de la calidad para garantizar que sus productos lleguen al cliente de forma que satisfaga plenamente sus expectativas, para este caso de investigación en particular que el producto cumpla con las regulaciones vigentes en lo que a distribución eléctrica se refiere.

En ese sentido, la distribución eléctrica es un área medular dentro de la industria eléctrica ya que es el punto de enlace con el cliente o consumidor tanto industrial como doméstico, este está sujeto expansiones continuas y cambios constantes en la cantidad de subscritores y potencia a suministrar, esto hace que el departamento de distribución eléctrica requiera mejoras continuas en la búsqueda de reducir costos y mejorar la productividad para continuar operando aun en momentos de alta demanda energética y ofrecer continuidad en el servicio dentro de las regulaciones estipuladas lo cual se ve definitivamente afectado.

La situación que se presenta en la industria de electricidad, igual que muchas otras de diversos sectores hoy día en Venezuela, se ha visto deteriorada por una depresión económica, las organizaciones se ven en la necesidad de aplicar estrategias relevantes para mantenerse en el tiempo y espacio. En el país las empresas de suministro y distribución de energía eléctrica antes operaban en bloques separados por estados, ahora han sido agrupadas en una sola empresa para todo el territorio nacional a partir del año 2001 con la entrada en vigencia de la Ley orgánica del servicio eléctrico y desde entonces lleva por nombre Corporación Eléctrica Nacional S.A. (CORPOELEC) empresa esta que no escapa a los efectos de la realidad antes mencionada en la cual está y sigue inmersa la Nación, es por eso que se hace necesaria la mejora continua de la gestión de la calidad de dicha empresa del sector eléctrico nacional.

La industria eléctrica nacional está sujeta a cambios constantes impulsada por factores como la creciente demanda, las condiciones ambientales e implementación de nuevas tecnologías las cuales han impactado de manera significativa la gestión en la distribución en la industria eléctrica, así mismo, el departamento de distribución eléctrica debe garantizar el buen manejo de la gestión de la calidad en todas sus redes eléctricas así como la prestación de un servicio óptimo por parte de la empresa CORPOELEC con el fin de satisfacer las necesidades de sus suscriptores y hacer los recursos organizacionales más rentables.

Es por ello y detectando la necesidad que tiene la empresa Corporación Eléctrica Nacional, 
C.A. (CORPOELEC) en su departamento de distribución eléctrica para todas sus divisiones, como lo son: operaciones, mantenimiento, recursos humanos y planificación, para realizar mejoras sustanciales en la calidad de entrega de su producto que no es más que la distribución de energía eléctrica al consumidor final tal y como lo define Reglamento General de Ley del Servicio Eléctrico Decreto $\mathrm{N}^{\circ}$ 1.124 Cap. IV artículo 22 "La actividad de distribución consiste en el transporte, transformación y entrega de electricidad a los usuarios del servicio eléctrico, mediante líneas e instalaciones diferentes a las utilizadas en la actividad de transmisión" sea este de uso industrial o residencial de manera satisfactoria, se realizara este estudio con el objeto de potenciar las oportunidades que tienen de hacer mejoras en la entrega del producto.

En muchos casos las circunstancias ambientales, políticas y sociales hacen extremadamente costoso el proceso de distribución eléctrica afectando la calidad y continuidad del suministro del producto dentro de los parámetros sometidos a regulación como lo es el rango de voltaje del suministro, la frecuencia de sistema y la continuidad del mismo, para regular los rangos del suministro de la energía eléctrica en Venezuela en lo concerniente a voltaje y frecuencia el Comité de Electricidad de Venezuela Codelectra y Covenin definen el rango operación y variación permisible de estos parámetros en el suministro del servicio eléctrico a través de la norma venezolana (Covenin 159:1997).

El funcionamiento de los activos que constituye la red de distribución eléctrica como lo son: subestaciones, transformadores de potencia líneas o tendidos aéreos y subterráneos de la empresa CORPOELC para distribuir y comercializar su producto que no es otro que la comercialización de energía eléctrica, afecta de manera directa a la empresa en sí misma y a las empresas cliente en su balance de ganancias y pérdidas, el inadecuado funcionamientos de estos activos repercute en la continua y sana operatividad del sistema generando fluctuaciones y cortes del servicio en el suministro de energía eléctrica que afectan al cliente y por ende la rentabilidad sus empresas. Por otra parte para CORPOELEC esta afectación genera serias consecuencias dentro de las que se pueden mencionar: Daño a equipos eléctricos por cortes en el servicio o bajas de tensión y disminución en el servicio prestado por lo que disminuye los montos en facturación. Ambas afectan sensiblemente sus ingresos, por lo que se torna indispensable controlar esta pérdida económica.

Como conclusión de estos dos hechos, daños a los equipos y baja en facturación, se puede inferir que carecen de una buena gestión en la distribución eléctrica lo cual ocasiona cortes del servicio, que no solo afectan significativamente a los hogares, sino a las instituciones académicas, de salud, al comercio en general, las empresas de servicio y además trastornan los procesos productivos de las industrias suscriptoras, generando pérdidas económicas reflejadas en poca 
producción afectando los índices de productividad y anulando los indicadores de gestión de la industria.

En la actualidad se observan continuas fallas en la distribución eléctrica las cual afectan la calidad de vida de la población y el desarrollo socioeconómico del país toda vez que el sector industrial también se ve afectado por los cortes en el suministro de energía eléctrica, por ello se considera de la más alta relevancia. También el tiempo de respuesta en la corrección de fallas en el suministro eléctrico una vez reportado por el suscriptor hasta que se restablece el suministro de electricidad se ha incrementado pasando de noventa minutos promedio hace un par de años atrás a un tiempo de nueve horas promedio desde el momento en que el suscriptor reporta la ausencia del servicio eléctrico hasta el momento en el que el suministro de electricidad es restablecido.

Las causas más comunes que se presentan como accionantes del retraso para solventar las fallas con la rapidez son: la carencia de unidades móviles especialmente equipadas para la ejecución del trabajo, la falta equipos de reemplazo y la falta de materiales, que sin duda afecta los índices de eficiencia y crea en el suscriptor pérdidas económicas sea este del sector residencial o industrial malestar en el suscriptor a la vez que entorpece el buen desarrollo y continuo de las actividades rutinarias y para CORPOELEC se convierte en producto no vendido.

Así, la gestión de mantenimiento, actualización o renovación de equipos y tecnologías y el suministro de materiales constituyen un problema creciente dentro de la industria debido al retraso en la entrega de los mismos por diversas razones dentro de las que destaca la centralización de la procura de materiales y equipos, habida cuenta que esta se ha centralizado en la Región Capital, el departamento de distribución eléctrica local está obteniendo de procura central una dilatada respuesta en la entrega de los suministros solicitados.

Una rutina planificada y eficiente de inspección, mantenimiento y renovación de los activos de distribución aunado a una potencia suficiente para la distribución y suministro de energía a subscritores es pieza fundamental del proceso, en consecuencia, el mantener los equipos de distribución eléctrica en perfectas condiciones y avalado con un mantenimiento preventivo lograría dar una respuesta satisfactoria a los clientes, contar siempre con un producto que este presentado de cara al consumidor final de manera óptima y perfecta para el disfrute del mismo y permitiría generar mayor expansión y desarrollo de nuevas oportunidades de negocios tanto para quien ofrece el producto como para quien lo consume otorgando un desarrollo excepcional en tiempos de crisis. Lo expresado en párrafos anteriores permite plantearse la siguiente interrogante: ¿Cómo es la gestión de la calidad en la distribución eléctrica de la empresa CORPOELEC en los municipios de 
la Costa Oriental del Lago del estado Zulia?

\section{CONSIDERACIONES METODOLÓGICAS}

La investigación es descriptiva, donde se recogió información de manera independiente de la variable gestión de la calidad, identificando las características de la misma, describiendo la situación tal y como fue observada en el proceso de la distribución eléctrica de la empresa CORPOELEC de los municipios de la Costa Oriental del Lago del estado Zulia. Mientras que se orientó hacia un diseño de campo, no experimental y transversal.

En cuanto a la población se consideraron los departamentos de distribución eléctrica ubicados de los municipios Cabimas, Simón Bolívar y Lagunillas de la Costa Oriental del Lago del estado Zulia. Para abordar esas unidades de análisis, se entrevistó a los gerentes, coordinadores de departamentos y jefes de área de los departamentos de distribución eléctrica a fin de conocer su opinión con respecto a la problemática abordada.

De manera que se consideraron como informantes claves dos gerente, ocho coordinadores de departamento y seis jefes de área correspondientes a la estructura organizativa del departamento de distribución eléctrica de CORPOELEC en los municipios antes indicados. En virtud de que la población es finita y de fácil acceso se tomó un censo poblacional. El cual consistió en incluir todos y cada uno de los elementos que componen la población.

La técnica utilizada para la recolección de datos, se empleó la observación mediante encuesta. Al respecto, se elaboró un instrumento auto-administrado, conformado por 77 ítems, con una escala tipo Likert, estructuradas por cinco alternativas donde las de mayor puntaje, (5): Siempre y (4): Casi siempre, se refieren a las respuestas positivas, seguidas de la opción a veces (3), indicativa de una postura neutra del encuestado, culminando con las de menor puntaje, (2) casi nunca y (1) nunca, relacionadas con las respuestas de tendencia negativa.

Para interpretar los resultados, se procedió a la construcción de un baremo, siguiendo el proceso descrito por Tapia (2007), para conocer el nivel alcanzado por el indicador partiendo de la puntuación de las escala de respuesta del instrumento de recolección de datos (1 a 5). Para ello se determinó un de clase $(0,80)$ al cual se sumó al valor mínimo de la escala de respuesta (1) restando una unidad, resultando el primer intervalo $(1-1,79)$ y así sucesivamente se fueron estableciendo los rangos hasta llegar a 5, por ser el valor máximo de la escala de respuesta. Este baremo fue utilizado para comparar la media aritmética de los indicadores, dimensiones y variable para calificar si se encontraban en niveles adecuados o inadecuados, facilitando la interpretación de los resultados 
(ver cuadro 1).

\section{Cuadro 1}

Baremo para la interpretación de la media aritmética

\begin{tabular}{|c|c|c|c|}
\hline Escala & Puntuación & Rango & \multirow{2}{*}{ Nivel Alcanzado } \\
\hline Siempre & 5 & $4,20-5,00$ & \multirow{2}{*}{ Adecuado } \\
\hline Casi siempre & 4 & $3,40-4,19$ & \\
\hline A veces & 3 & $2,60-3,39$ & \multirow{2}{*}{ Inadecuado } \\
\hline Casi nunca & 2 & $1,80-2,59$ & \\
\hline Nunca & 1 & $1,00-1,79$ & \\
\hline
\end{tabular}

Fuente: Elaboración propia.

En lo que respecta a la validez de contenido del instrumento, este se realizó a través de la apreciación por parte de expertos de la correcta construcción del mismo, en cuanto a la redacción de los ítems y su congruente relación con los objetivos, dimensiones e indicadores de la variable de estudio. Para lograr esto, el cuestionario fue validado por un grupo de cinco expertos, empleando para ello formatos de validación especialmente diseñados por el investigador. Una vez recolectados los instrumentos validados, se cumplieron las sugerencias y recomendaciones en cuanto a mejorar la redacción de los ítems, eliminar aquellos que de acuerdo con el juicio de expertos no sean necesarios, o bien simplificarlos. Tras este proceso, se obtuvo una versión mejorada de los mismos y válida desde el punto de vista de su contenido.

En cuanto a la confiabilidad, se aplicó una prueba piloto, la cual consistió en aplicarlo a una población con características similares a las estudiadas (personal del departamento de distribución eléctrica). Los datos obtenidos fueron procesados para determinar la confiabilidad a través de la formula Alfa Cronbach, cuyo valor para el instrumento dirigido al personal del departamento de distribución eléctrica de los municipios Cabimas, Simón Bolívar y Lagunillas fue de 0,968, determinándose que es mismo es confiable.

Para efectuar el análisis estadístico, los datos fueron ordenados, codificados y tabulados, luego de la aplicación de la versión final del instrumento a la población del estudio. Luego procedió a presentar las dimensiones e indicadores de la variable a través de la estadística descriptiva, calculando frecuencias absolutas y relativas. Posteriormente, estos resultados se representaron en tablas de doble entrada para una mayor comprensión, discusión y análisis. 


\section{PRESENTACIÓN Y DISCUSIÓN DE LOS RESULTADOS \\ Procesos en el sistema de gestión de la calidad en el departamento de distribución eléctrica de la empresa CORPOELEC}

La norma ISO 9000:2005 establece que un sistema de gestión de calidad (SGC) es un sistema diseñado para dirigir y controlar una organización con respecto a la calidad. También puede asumirse que es aquella parte del sistema de una organización enfocada en el logro de los resultados, en relación a los objetivos de calidad, para satisfacer las necesidades, expectativas y requisitos de las partes interesadas. Es por tanto, un ordenamiento sistemático de todas las acciones necesarias, que abarca la estructura organizativa, responsabilidades, recursos necesarios, procesos y procedimientos.

En el mismo orden de ideas, Cuatrecasas (2010) plantea que un sistema de gestión de la calidad tiene como objetivo básico conseguir plenamente la calidad necesaria expresada por los clientes. Para ello ha de procurar llevar a cabo la correcta gestión de todos los procesos relacionados con la calidad; esto incluye la planificación, diseño y desarrollo tanto de productos como procesos en el marco de una organización y gestión de recursos humanos para la calidad, atendiendo a su adecuada implantación, control y certificación final. Un sistema de gestión de la calidad así concebido, llevará la empresa a obtener el máximo de ventajas competitivas y la satisfacción total de los clientes mediante la identificación, aceptación y cobertura de sus necesidades. Según lo plantea el autor, en la gestión orientada a la calidad es el propio cliente quien determina el grado de calidad que precisa.

Ampliando las ideas anteriores, Alcalde (2009: 78) define un sistema de gestión de calidad como "un conjunto de procesos mutuamente relacionados o que interactúan para establecer la política y los objetivos con el que dirigir y controlar la organización con respecto a la calidad". Al respecto, el autor refiere que la norma ISO 9001:2008, especifica los requisitos para un sistema de gestión aplicable a toda organización que necesite demostrar su capacidad para proporcionar los productos que cumplan los requisitos de sus clientes, así como con los reglamentarios que regulan su actividad, todo con el interés de aumentar la satisfacción del cliente.

\section{a) Responsabilidad de la Dirección.}

La Norma ISO 9001: 2008, establece que la dirección tendrá la responsabilidad de: a) diseñar e implantar del sistema de gestión de calidad, b) asegurar un enfoque hacia la satisfacción del cliente, c) definir la política de calidad, así como establecer los niveles tanto de responsabilidad, como de autoridad para la toma de decisiones, f) revisar el cumplimiento del sistema de la calidad.

Por otra parte, Nava y Jiménez (2005), especifican que la participación de la alta dirección 
es uno de los factores más importantes en el desarrollo del sistema de calidad. Básicamente, las responsabilidades específicas de la dirección tienen que ver con la identificación de necesidades del cliente, desarrollo de la política y objetivos de calidad, así como la planeación, asignación de recursos y revisión de la efectividad del sistema.

Según estos autores, es esencial que la dirección establezca un sistema de comunicación interna que permita al resto del personal, estar consciente del alcance del sistema y de cómo su participación es determinante para el logro de los objetivos. Asimismo, la dirección debe hacer que se conozca en toda la organización lo importante que es cumplir con los requisitos del cliente para la supervivencia de la empresa, así como con los legales y reglamentarios que aplican a su área de actividad. En este sentido, en la tabla 1 se evidencian los indicadores abordados: Compromiso de la Dirección, política de calidad, planificación y comunicación interna, obteniéndose en global una media de 3,98, situando a esta categoría de análisis en un nivel adecuado.

Ya en detalle, se presenta en primer lugar el compromiso de la dirección, en la misma tabla 1 se observa que alcanzó un valor de 4,09 quedando ubicado en un nivel adecuado al ser comparado dicho valor con el baremo diseñado por el investigador para cualificar las medias aritméticas en la presente investigación. Se evidencia que en el departamento de distribución eléctrica de los municipios Cabimas Simón Bolívar y Lagunillas prevalece el compromiso de la dirección para estimular e impulsar al resto del personal al alcance de la calidad total (siempre 37,5\% y casi siempre 50\%).

Es muy difícil sin ese compromiso formal y efectivo, conseguir la participación de todos los miembros de la empresa para alcanzar la eficacia del sistema de calidad. En este sentido, la dirección establece la política de calidad y se asegura de que sea internalizada por todos los niveles organizativos, de forma que se demuestren evidencias de su puesta en práctica por todos los empleados (siempre 43,8\% y casi siempre 31,3\%). En definitiva, sin el compromiso de la dirección es improbable que el sistema de gestión de calidad sea operativo.

Tabla 1. Responsabilidad de la dirección

\begin{tabular}{|c|c|}
\hline Indicadores & Media de los indicadores \\
\hline $\begin{array}{c}\text { Compromiso de la } \\
\text { Dirección }\end{array}$ & 4.09 \\
\hline Política de calidad & 4.02 \\
\hline Planificación & 4.04 \\
\hline Comunicación interna & 3.75 \\
\hline Media & 3.98 \\
\hline
\end{tabular}

Fuente: Elaboración propia 
Con respecto a la Política de Calidad, los resultados obtenidos, muestran en la tabla 1, que la media para el indicador fue de 4,02 quedando ubicada en un nivel adecuado. Esto como consecuencia que las respuestas siempre y casi siempre suman el 68,8\% de la preferencia como respuesta para los encuestados. De manera que para los informantes claves, la gerencia se asegura que la política de la calidad sea adecuada al propósito del departamento de distribución eléctrica; las actividades que permiten implementar la política de la calidad son controladas para su mejora de la calidad continua y en el departamento de distribución eléctrica la política de la calidad está acorde a las necesidades del cliente.

Al respecto, la norma ISO 9001: 2008, indica que la alta dirección debe asegurarse de que la política de la calidad sea adecuada al propósito de la organización e incluya el compromiso de cumplir con los requisitos y de mejorar continuamente la eficacia del sistema de gestión de la calidad, Esta política, debe proporcionar un marco de referencia para establecer y revisar los objetivos a alcanzar, por tanto es determinante que sea comunicada y entendida dentro de la organización, así como revisada para su mejora continua.

En lo que corresponde a la media del indicador planificación de la calidad, ésta alcanzó un valor de 4,04 quedando ubicado en un nivel adecuado (ver tabla 1). Este resultado, permite señalar que la gerencia del departamento en estudio planifica el sistema de gestión de la calidad con el fin de cumplir los siguientes aspectos:(a) Declaraciones documentadas de la política y objetivos de la calidad (siempre y casi siempre 68,8\%). (b) Constituir un manual de la calidad (siempre y casi siempre 68,8\%). Y (c) Documentar los procedimientos a seguir: planificación, operación y control de sus procesos, para el alcance de los objetivos (siempre y casi siempre 81,3\%). Asimismo, la norma establece que la planificación debe mantener la integridad del sistema de gestión de la calidad cuando se planifican e implementan cambios.

A propósito de la comunicación interna, la diversidad en las repuestas de los entrevistados denota cierta debilidad en la comunicación dentro del departamento, sin embargo la media del indicador se ubicó en el extremo inferior obteniendo un valor de 3,75 correspondiéndole un nivel adecuado (ver tabla 1). Este resultado refleja que en el departamento de distribución eléctrica la comunicación involucra adecuadamente a todo el personal haciendo que este se sienta parte del sistema de gestión de la calidad (50,1\% siempre y casi siempre) y la gerencia realiza reuniones de trabajo con el personal para comunicarles los problemas que se han presentado en la implementación de la política de la calidad (62,6\% siempre y casi siempre.

De acuerdo a Nava y Jiménez (2005), la adecuada comunicación interna es la base para el 
entendimiento de los objetivos de calidad por parte de todo el personal., además elimina barreras permitiendo que el personal se involucre adecuadamente en el trabajo en equipo, sintiéndose parte activa del sistema de gestión de calidad.

\section{b) Gestión de recursos.}

La norma ISO 9001:2008, establece que la empresa para implementar de forma eficiente el sistema de gestión de calidad debe gestionar los recursos necesarios para la obtención del producto o servicio solicitado por el cliente, dentro de lo cual incluye: Provisión de recursos, recursos humanos, infraestructura o instalaciones y ambiente de trabajo.

Tabla 2. Gestión de recursos

\begin{tabular}{|c|c|}
\hline Indicadores & Media de los indicadores \\
\hline Provisión de recursos & 3.04 \\
\hline Recursos Humanos & 4.30 \\
\hline Infraestructura & 3.63 \\
\hline Ambiente de trabajo & 3.07 \\
\hline Media & 3.51 \\
\hline
\end{tabular}

Fuente: Elaboración propia.

En la tabla 2 se agrupan los resultados obtenidos para la dimensión gestión de recursos, como se puede apreciar, la media alcanza un valor de 3,51 que lo ubica a un nivel adecuado pero situado en el extremo inferior del baremo establecido. Los resultados para los indicadores: recursos humanos e infraestructura, apenas fueron suficiente para alcanzar un nivel de adecuado de la media alcanzada.

De manera que en función a los resultados obtenidos se puede indicar que el departamento de distribución eléctrica de CORPOELEC de los municipios Cabimas, Simón Bolívar y Lagunillas deben realizar mayores esfuerzos para implementar de forma eficiente el sistema de gestión de calidad, gestionando eficientemente los recursos necesarios para la obtención del producto o servicio solicitado por el cliente.

En detalle, en cuanto a la provisión de los recursos, los datos obtenidos muestran que el $81,3 \%$ de los encuestados manifestaron que el departamento de distribución eléctrica no cuenta con inventario suficiente de repuestos para atender averías del sistema, cualificando este aspecto como inadecuado, con una media de 2,38. En tanto, un $68,8 \%$ manifestó que disponen de equipos y unidades especializadas para satisfacer las necesidades de los suscriptores. Estos resultados califican a la provisión de recursos en un nivel inadecuado $(3,04)$. 
Para Nava y Jiménez (2005), la dirección conjuntamente con el equipo responsable del Sistema de Gestión de la Calidad (SGC) deben responder a las siguientes interrogantes: ¿Qué recursos son necesarios para comunicar los requisitos del cliente y la importancia de cumplir con ellos? ¿Qué recursos son necesarios para abordar las quejas del cliente? El equipo de revisión de la dirección en todo caso, debe preguntarse si están disponibles los recursos necesarios para desarrollar las acciones propuestas. En este sentido, el departamento de distribución eléctrica está presentando fallas en la respuesta de una o varias de estas interrogantes trayendo como consecuencia un pobre desempeño en la reposición de inventarios y la reparación y/o reposición de equipos y unidades especializadas.

En otro orden de ideas, de acuerdo con la opinión del personal del departamento de distribución eléctrica, se encuentra profesionalmente capacitado para el óptimo desempeño de sus funciones; posee registros apropiados del nivel de formación de su personal; proporciona formación para lograr que el personal posea todas las competencias necesarias inherentes al cargo y el personal es consciente de la importancia de sus actividades para el logro de los objetivos de la calidad. De manera que la media del indicador recursos humanos se ubicó en 4,30 correspondiéndole un nivel adecuado.

Para Cuatrecasas (2010), los recursos humanos representan un papel esencial en el desarrollo y obtención de los objetivos de calidad, dado que es imposible lograrlos si no existe una clara motivación de todos los estamentos que forman la empresas, con entusiasmo y con la convicción plena de que es la mejor forma para satisfacer plenamente a los clientes y alcanzar un alto grado de excelencia. La calidad es tarea de todos y su implicación va a depender de una correcta selección del personal que mediante un proceso de formación adecuado, trabaje con criterios acordes con la cultura de calidad establecida en la empresa.

Con respecto a la Infraestructura, se obtuvo un valor de 3,63 correspondiéndole un nivel adecuado en la empresa objeto de estudio al compararlo con el baremo del investigador. Su baja ponderación se debió a que el 62,6\% de los entrevistados opinaron que el departamento de distribución eléctrica no cuenta con infraestructura (edificaciones, espacios de trabajo y servicios asociados) necesaria para el trabajo a realizar. En contraposición el 81,3\%, señaló que el departamento de distribución eléctrica posee equipos informáticos para llevar a cabo los procesos.

Estos resultados permiten inferir que se presentan serias debilidades para cumplir con los requisitos para la gestión de la calidad. Al respecto, la norma ISO 9001:2008, señala que la organización debe determinar, proporcionar y mantener la infraestructura necesaria para lograr 
la conformidad con los requisitos del producto. La infraestructura incluye, cuando sea aplicable: (a) Edificios, espacio de trabajo y servicios asociados. (b) Equipos informáticos para los procesos (tanto hardware como software). (c) Servicios de apoyo (tales como transporte, comunicación o sistemas de información).

Al abordar el Ambiente de trabajo, este indicador se ubicó en un valor inadecuado de 3,07. Se observa que la mayor parte de los encuestados $(75,1 \%)$, coincidieron en sus respuestas con orientación negativa, al señalar que en el departamento de distribución eléctrica no se cuenta con adecuadas condiciones ambientales que permiten confort en el desempeño laboral. De igual manera, un alto porcentaje $(56,3 \%)$ señaló que no se gestiona el ambiente de trabajo para lograr la conformidad con los requisitos del producto.

En este sentido la norma ISO 9001:2008, es explicita al señalar que la organización debe determinar y gestionar el ambiente de trabajo necesario para lograr la conformidad con los requisitos del producto. El término ambiente de trabajo está relacionado con aquellas condiciones bajo las cuales se realiza el trabajo, incluyendo factores físicos, ambientales y de otro tipo (tales como el ruido, la temperatura, la humedad, la iluminación o las condiciones climáticas). El alto porcentaje de repuestas adversas, dadas por los entrevistados, permite inferir el incumplimiento por parte de la gerencia de la Distribución Eléctrica de la empresa CORPOELEC en la gestión inherente a un adecuado ambiente de trabajo para el sano desempeño de las actividades laborales de su personal.

\section{c) Mejora continua del sistema de administración por calidad.}

La norma ISO 9001:2008 establece que la organización debe mejorar continuamente la eficacia del sistema de gestión de la calidad mediante el uso de la política de la calidad, los objetivos de la calidad, los resultados de las auditorías, el análisis de datos, las acciones correctivas/ preventivas y la revisión por la dirección. Al respecto, en la tabla 3, se observa la media para la mejora continua del sistema de administración por calidad, alcanzando un valor de 4,50 que la ubica a un nivel adecuado.

Los resultados demuestran que todos los entrevistados coinciden en afirmar que se aplican acciones preventivas para eliminar causas de no conformidades o fallas potenciales para prevenir su ocurrencia. Y el 93,8\% piensa que en el departamento de distribución eléctrica se revisa la eficacia de las acciones preventivas tomadas. De esta forma cumplen lo establecido en la norma ISO 9001:2008, en cuanto a eliminar las causas de no conformidades potenciales previniendo su ocurrencia, seleccionar medidas preventivas apropiadas a los efectos de los problemas potenciales, evaluar la necesidad de actuar para prevenir la ocurrencia de no conformidades, registrar los 
resultados de las acciones aplicadas y revisar la eficacia de las medidas preventivas tomadas.

Tabla 3. Mejora continua del sistema de administración por calidad

\begin{tabular}{|r|c|}
\hline Indicadores & Media de los indicadores \\
\hline Acción preventiva & 4.50 \\
\hline Acción correctiva & 4.50 \\
\hline Media & 4.50 \\
\hline
\end{tabular}

Fuente: Elaboración propia

Igual comportamiento se observa en los resultados obtenidos en cuanto a las acciones correctivas, donde un alto porcentaje $(93,8 \%)$ afirma que en el departamento de distribución eléctrica, las acciones correctivas son apropiadas para eliminar las causas de las no conformidades o fallas encontradas y se revisa la eficacia de las acciones correctivas tomadas.

Culminado los resultados de los Procesos del sistema de gestión de la calidad, se puede señalar que esta dimensión alcanzó un valor adecuado de 4.01. Valor obtenido en promedio de la Responsabilidad de la dirección (4.02); gestión de los recursos (3.51) y mejora continua del sistema de administración por calidad (4.50). En este sentido se puede admitir que el departamento de distribución eléctrica está enfocado al logro de los resultados, en relación a los objetivos de la calidad, para satisfacer las necesidades, expectativas y requisitos de las partes interesadas.

\section{Principios de gestión de la calidad.}

De acuerdo con Camisón y col. (2007), los principios de gestión de la calidad permiten dar un seguimiento y evaluación permanente de los clientes, con el propósito de obtener información de sus necesidades, la evolución de sus requerimientos, su satisfacción o reacción ante el servicio recibido. Esta información es importante para compartir con los miembros de la empresa la definición de calidad exigida por los clientes.

De manera que los principios de gestión de calidad para Camisón y col. (2007) son: Orientación al cliente, orientación a la creación de valor, Liderazgo y compromiso de la dirección, orientación a las personas y desarrollo de competencias, visión sistémica de la organización, orientación a la cooperación, orientación al aprendizaje e innovación y la orientación ética y social, los cuales se presentan a continuación:

\section{a) Orientación al cliente.}

Este principio de calidad postula la consideración del cliente como el eje de la actividad 
empresarial, la cual debe enfocarse a su satisfacción, tal como lo indican Camisón y col. (2007). Esto es debido, a que los clientes son quienes tienen la potestad de enjuiciar la calidad del servicio prestado por la empresa. Por tal razón, la empresa debe cambiar su enfoque de orientar la calidad hacia el producto para orientarla hacia el cliente, pues todas las actividades empresariales están alineadas a servir a un cliente que no sólo busca un producto de calidad, sino que además exige información, asesoramiento, servicio a tiempo, garantía de calidad, entre otros.

$\mathrm{Al}$ respecto, la media para el indicador orientación al cliente alcanzó un valor adecuado de 3,97. Dato obtenido al observar las respuestas dadas por los entrevistados. Donde, el $73 \%$ respondió positivamente al afirmar que en el departamento de distribución eléctrica se realiza seguimiento de las necesidades de los suscriptores y para el $68,8 \%$ se satisfacen las necesidades del cliente en cuanto al tiempo de respuesta ocasionado por reclamos ante una desviación en la distribución eléctrica.

\section{b) Orientación a la creación de valor.}

La orientación estratégica hacia la creación de valor exige la aplicación de distintas prácticas que garanticen la integración de la calidad en el proceso estratégico de la empresa, entre las cuales se pueden mencionar: la investigación e identificación de necesidades de los grupos de interés clave de la organización, la enunciación de objetivos e indicadores de desempeño, las prácticas enfocadas a explorar el potencial de creación de ventajas competitivas que encierran la calidad y su gestión, las prácticas enfocadas a la cooperación tanto con clientes como con proveedores para la mejora continua de la calidad, las prácticas de orientación hacia la innovación, así como las prácticas organizativas que estimulen el alcance de activos valiosos como fuentes de ventajas competitivas (Camisón y col. 2007).

Al calcular la media para el indicador orientación a la creación de valor, ésta alcanzó un nivel adecuado de 3,88. Así, se puede observar que al aplicar el instrumento, el 56,3\% de los entrevistados manifestó que el departamento de distribución eléctrica siempre o casi siempre posee estrategias corporativas que integran la calidad en el proceso de formulación e implantación estratégica. De igual manera, un alto porcentaje $(68,8 \%)$ respondió afirmativamente que la calidad en la distribución eléctrica se encuentra incluida como principio en la planificación estratégica del departamento de distribución eléctrica. Además, queda de manifiesto que la mayoría de los encuestados $(68,8 \%)$ respondió que siempre o casi siempre la calidad en la distribución eléctrica se encuentra incluida como principio en la planificación estratégica del departamento de distribución eléctrica mientras que el restante $31,3 \%$ respondió a veces o casi nunca al planteamiento. 


\section{c) Liderazgo y compromiso de la dirección.}

Cuatrecasas (2010), considera al liderazgo como una característica de la calidad y su gestión, basado en la importancia del aporte de la dirección en la implantación efectiva de la calidad. Para el autor, la gestión de la calidad debe contar con todo el apoyo y liderazgo de la alta dirección, la cual a su vez, debe implicarse practicando con el ejemplo en la consecución de los objetivos de la calidad de forma activa. Esto requiere un estilo de gestión participativa que promueva un consenso en la toma de decisiones, con la intervención de los miembros de la empresa.

De manera que, los resultados revelan que el $75 \%$ del personal encuestado opinan que siempre los líderes del departamento de distribución eléctrica orientan al personal hacia el logro de los objetivos. De igual forma, la mayoría de los encuestados (87,5\%), coincidió en responder que siempre y casi siempre el líder promueve el consenso en la toma de decisiones con la intervención de todos los miembros de la empresa. Estos resultados llevan a obtener una media para el indicador de 4,47, siendo calificada como adecuada.

\section{d) Orientación a las personas y desarrollo de competencias.}

De acuerdo a Camisón y col. (2007), este principio se encuentra íntimamente relacionado con el compromiso y liderazgo de la dirección, la visión global de la empresa y la cooperación interna. Asimismo, establece que para ver los frutos de la gestión de la calidad, la organización debe desplegar conocimientos, habilidades, responsabilidad y compromiso con el fin de inducir la conciencia de la calidad en sus miembros, partiendo de que a calidad se hace con las personas en vez de hacerse a las personas. Se fundamenta en la creencia de que los miembros de la organización distintos a los directivos, son los que mejor conocen el trabajo, pudiendo realizar importantes contribuciones a la mejora continua y satisfacción de los clientes, cuando obtienen el potencial requerido, esto implica compromiso, participación, desarrollo de competencias y formación.

Al respecto, los resultados evidencian que los encuestados presentan opiniones divididas toda vez que el $50 \%$ respondió siempre o casi siempre al consultar si la gerencia del departamento posee programas de desarrollo de competencias y capacitación del personal mientras el 50\% respondió a veces, casi nunca y nunca. También se demuestran que la mayoría de los encuestados (62,5\%) manifestó respuesta con orientación negativa, al señalar que en el departamento de distribución eléctrica no se desarrollan sistemas de reconocimiento que incorporen recompensas al esfuerzo y logro de metas propuestas. La media para el indicador orientación a las personas y desarrollo de competencias, fue de 3,16, siendo calificado como inadecuado lo cual evidencia que en el departamento estudiado no se toma en cuenta la orientación hacia las personas como un principio que sustenta le gestión de la calidad. 


\section{e) Visión sistémica de la organización.}

La visión global de la gestión de calidad moderna exige la participación de todos los miembros y todas las funciones de la organización orientadas a satisfacer al cliente, así como a la reducción de costes que no añaden valor. Por tanto, todas las unidades funcionales tienen el deber de identificar, controlar y mejorar los grados de calidad dentro de su área de responsabilidad. Esta visión global sostiene la práctica de la gestión por procesos y en un pensamiento sistémico. Esta perspectiva sistémica permite una visión holística de la gestión de procesos y personas, provee una plataforma adecuada para estructurar cambios culturales y organizativos precisados por la gestión de la calidad (Camisón y col.2007).

En este sentido el $62,5 \%$ de los entrevistados piensan que siempre o casi siempre los miembros del departamento de distribución eléctrica participan en todas las funciones de la organización orientándola a satisfacer al cliente. Mientras que el 75\% manifestó que siempre y casi siempre en el departamento existe una visión global de la gestión de procesos adecuada para propiciar cambios culturales y organizativos requeridos por la gestión de la calidad. Estos resultados concretaron una media adecuada de 3,63 .

\section{f) Orientación a la cooperación.}

La cooperación debe ser tanto interna como externa para generar frutos positivos sobre el aprendizaje y la capacidad de adaptación e innovación de los socios. Compartir recursos, conocimientos, información o experiencias facilita el enriquecimiento y la renovación del stock de competencias. Además, la cooperación facilita la experimentación con nuevas formas de coordinación más perfeccionadas, que coadyuven al desarrollo de la capacidad de adaptación, de la flexibilidad y de la reducción de los tiempos de respuestas a los cambios. Este principio se lleva a cabo por medio del establecimiento de alianzas estratégicas con proveedores, establecer equipos conjuntos con los clientes haciéndolos partícipes en el diseño del producto y la aplicabilidad de programas de mejora de los proveedores tipo calidad acertada que permitan intercambiar conocimientos, información, experiencias, así como el desarrollo de nuevas competencias, para aprender a gestionar y mejorar la calidad de sus procesos (Camisón y col 2007).

Los resultados indican que $87,6 \%$ de los encuestados estuvieron de acuerdo que siempre y casi siempre en el departamento se comparten recursos, conocimientos, información o experiencias que facilite el enriquecimiento de las competencias. En tanto, para 62,5\%, respondió negantemente al señalar que en el departamento de distribución eléctrica no se establecen alianzas estratégicas con proveedores y clientes para hacerlos partícipes en la gestión y mejora de la calidad de sus procesos. Sin embargo, la media para este principio fue de 3,51, ubicándose en un nivel adecuado. 


\section{g) Indicador: Orientación al aprendizaje e innovación.}

Este principio postula que la dirección debe liderar la organización dando la oportunidad de implantar el aprendizaje adaptativo y generativo (mejora continua), así como la innovación incremental y radical (mejora radical), según lo indican Camisón y col. (2007). La orientación hacia el aprendizaje solo es sostenible con la participación y compromiso de los miembros de la organización, aunado a la visión de excelencia de la dirección. La eficacia del aprendizaje descansa en el enriquecimiento de los conocimientos de los empleados y directivos mediante acciones de formación.

Los resultados permiten obtener una media de 4,35 siendo valorado como adecuado. Este valor se obtuvo de las respuestas dadas por los entrevistados quienes respondieron $(93,8 \%)$ que siempre y casi siempre, el departamento de distribución eléctrica posee un sistema de calidad que documenta los procesos, procedimientos, e instrucciones de trabajo que estandarizan y sistematizan el trabajo. Y el 93,8\% respondió afirmativamente que departamento cuenta con indicadores de evaluación de los procesos que faciliten la comunicación.

\section{g) Orientación ética y social.}

La gestión de la calidad no sólo está dirigida a las actividades internas de las empresas, sino a los efectos que dichas actividades tienen sobre otros agentes externos como clientes, proveedores, comunidad local, instituciones públicas, así como a la sociedad en conjunto, tal como lo señalan Camisón y col. (2007). Por tal razón, surge este principio de orientación ética y social, el cual trata un planteamiento próximo a los enfoques de responsabilidad social corporativa y ética empresarial.

En este sentido la media para este principio fue de 3,69 siendo valorado como adecuado. En detalle los resultados indican que el $68,8 \%$ de los encuestados estuvieron de acuerdo con que siempre y casi siempre se evalúan los efectos que producen sus actividades sobre otros agentes como son clientes, proveedores y la comunidad. Mientras que el 62,6\% estuvo de acuerdo en que siempre y casi siempre en el departamento distribución eléctrica se mide y corrige el impacto ambiental causado por sus procesos. En tanto el $56,3 \%$ opina que siempre y casi siempre se previene el deterioro de los recursos naturales causado por sus procesos.

Resumiendo lo expresado en párrafos anteriores, se determina que la media para los principios de gestión de la calidad se ubicó en 3,83 indicando nivel adecuado. Sin embargo se observa debilidades en la mayoría de sus componentes, ya que la media se ubica en el extremo inferior del nivel adecuado. De manera, en términos generales, se hacen esfuerzos para mantener la orientación de la gerencia hacia los clientes; se establecen sistemas de liderazgo que promueven el 
desarrollo de una cultura de participación; poco involucramiento, trabajo en equipo y planeación de las personas para desarrollar sus competencias; se orienta la organización hacia el aprendizaje e innovación, todo con una orientación ética y social que le permite mantenerse competitiva (ver tabla 4).

Tabla 4. Principios de gestión de la calidad

\begin{tabular}{|l|c|}
\hline \multicolumn{1}{|c|}{ Indicadores } & Media indicadores \\
\hline Orientación al cliente & 3.97 \\
\hline Orientación a la creación de valor & 3.88 \\
\hline Liderazgo y compromiso de la dirección & 4.47 \\
\hline $\begin{array}{l}\text { Orientación a las personas y desarrollo } \\
\text { de competencias }\end{array}$ & 3.16 \\
\hline Visión sistémica de la organización & 3.63 \\
\hline Orientación a la cooperación & 3.51 \\
\hline Orientación al aprendizaje e innovación & 4.36 \\
\hline Orientación ética social & 3.69 \\
\hline \multicolumn{1}{|r|}{ Media } & 3.83 \\
\hline
\end{tabular}

Fuente: Elaboración propia.

\section{A MANERA DE CONCLUSIÓN}

Se puede observar que la media de la variable gestión de la calidad, considerando los procesos del sistema de gestión de la calidad $(4,01)$, los principios de gestión de la calidad $(3,83)$, alcanzó una media de 3,92 que ubica a la variable en un nivel adecuado al ser comparado con el baremo diseñado para tal fin.

Es notorio que el departamento de distribución eléctrica de los municipios Cabimas, Simón Bolívar y Lagunillas del estado Zulia, CORPOELEC asume de manera adecuada la gestión de la calidad como una filosofía de dirección, orientada al cumplimiento de los principios de la calidad. Sin embargo, algunos aspectos deben ser atendidos para cumplir de conformidad con los requisitos o especificaciones establecidas por los clientes; debido a que están muy cercanos al extremo inferior de la media ponderada, lo cual alerta a prestar especial atención ya que puede ser susceptible a descender a valores inferiores. 


\section{BIBLIOGRAFÍA}

- Alcalde, P. (2009) Calidad. 1ra. Edición. España. Editorial Thompson Paraninfo.

- Camisón, C.; Cruz, S. y González, T. (2007) Gestión de la calidad: Conceptos, enfoques, modelos y sistemas. España. Pearson Educación.

- Cantú, H. (2006) Desarrollo de una cultura de calidad. México. McGraw Hill Interamericana Editores, S.A. de C.V.

- Cuatrecasas, L. (2010) Gestión integral de la calidad. Implantación, control y certificación. España. Profit Editorial.

- Evans, J., Lindsay, W. (2007) Administración y control de la calidad. Séptima edición. México. Editorial CengageLearning.

- Nava, V., Jiménez, A. (2005) Estrategias para implantar la Norma de Calidad para la Mejora Continua. México. Editorial Limusa Noriega Editores.

- Norma Venezolana FONDONORMA ISO 9000:2005. Sistemas de gestión de la calidad. Fundamentos y vocabulario. Comité Técnico de Normalización FONDONORMA CT23 Gestión de la Calidad aprobada por FONDONORMA Consejo Superior N ${ }^{\circ}$ 2005-02 de fecha 26/04/2006

- Organización Internacional para la Normalización (2001) Norma ISO 9000. Ginebra Suiza. Disponible en: http://sisbib.unmsm.edu.pe/bibvirtualdata/publicaciones/indata/vol4_2/a07. pdf. Consultado: 6/10/2018

- Organización Internacional para la Normalización (2005) Norma ISO 9000. Ginebra Suiza. Disponible en: http://www.uco.es/sae/archivo/normativa/ISO_9000_2005.pdf. Consultado: $6 / 10 / 2018$

- Organización Internacional para la Normalización (2008) Norma ISO 9000 (2008). Disponible en: http://farmacia.unmsm.edu.pe/noticias/2012/documentos/ISO-9001.pdf. Consultado: $6 / 10 / 2018$

- Tapia, J. (2007). Introducción al Análisis de Datos con SPSS para Windows. Venezuela. Ediciones Universidad Ezequiel Zamora. 